\title{
Misdiagnosis of pathological femoral fracture in a patient with intramuscular hemangioma: A case report
}

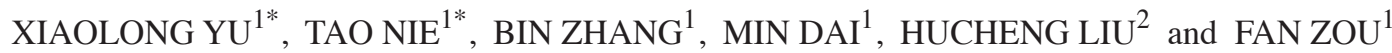 \\ ${ }^{1}$ Department of Orthopedics, The First Affiliated Hospital of Nanchang University, Artificial Joints Engineering and \\ Technology Research Center of Jiangxi Province; ${ }^{2}$ Multidisciplinary Therapy Center of Musculoskeletal Tumor, \\ The First Affiliated Hospital of Nanchang University, Nanchang, Jiangxi 330006, P.R. China
}

Received March 7, 2015; Accepted April 1, 2016

DOI: $10.3892 / \mathrm{ol} .2016 .4610$

\begin{abstract}
Hemangioma is a common disease; however, intramuscular hemangioma ( $\mathrm{IH})$ presenting with a pathological fracture is extremely rare. The present study reports a case of a 46-year-old male patient that suffered from IH of the right thigh, presenting with a pathological femoral fracture. The patient was initially diagnosed with a traumatic femoral fracture, and routine open reduction and internal fixation were performed at a local hospital. However, 20 days subsequent to surgery, gradual swelling and soreness around the incision were observed. The incision eventually ruptured during squatting for bowel movement, which led to extensive blood loss. Based on computed tomography (CT) and deep femoral artery arteriography, IH presenting with a pathological femoral fracture was diagnosed. The patient underwent artery embolization, from which he recovered well. At the 6-month follow-up, the femoral fracture was revealed to have healed, and a CT scan demonstrated no evidence of recurrence; however, continuous observation using $\mathrm{CT}$ is required in order to determine the long-term outcome. To the best of our knowledge, this is the first case of a misdiagnosed pathological femoral fracture in a patient with IH reported in the English literature.
\end{abstract}

\section{Introduction}

Hemangioma is a common benign vascular tumor usually observed in the liver, skin, eyes and central nervous system. However, intramuscular hemangiomas (IHs) are extremely rare, accounting for $<1 \%$ of all hemangiomas (1). IH frequently

Correspondence to: Mr. Fan Zou, Department of Orthopedics, The First Affiliated Hospital of Nanchang University, Artificial Joints Engineering and Technology Research Center of Jiangxi Province, 17 Yong Wai Zheng Street, Nanchang, Jiangxi 330006, P.R. China E-mail: 1207672246@qq.com

${ }^{*}$ Contributed equally

Key words: misdiagnosis, femoral fracture, arteriography, artery embolization, intramuscular hemangioma, ORIF occurs in the extremities, particularly the lower limbs. Since the majority of lesions are small, isolated and asymptomatic, the true incidence of hemangioma is very difficwult to calculate (2). Chronic pain and novel mass development comprise the most common symptoms of IH and hemangioma in general (3). Diagnostic methods for $\mathrm{IH}$ and hemangioma include clinical and laboratory examination, plain radiographs, ultrasound, computed tomography (CT), angiography and magnetic resonance imaging (MRI) (4). There are several treatment methods available for $\mathrm{IH}$ and hemangioma, including conservative therapy, systemic corticosteroids, embolization, radiation, sclerotherapy and surgical excision. Treatment for IH and hemangioma should be individualized (5). In the present study, the patient was mistakenly diagnosed with a common traumatic femoral fracture. The missed diagnosis of IH led to the rupture of an incision following open reduction and internal fixation (ORIF), which was accompanied by extensive blood loss. A careful review of the patient's history and detailed clinical examination are important for the identification of fractures, particularly in cases without a definite history of trauma. In the present study, CT and arteriography confirmed the diagnosis of pathological femoral fracture with $\mathrm{IH}$; however, the misdiagnosis of the patient's condition resulted in a markedly high cost and suffering for the patient. The present study was approved by the Ethics Review Committee of The First Affiliated Hospital of Nanchang University (Nanchang, China), and written informed consent was obtained from the patient.

\section{Case report}

A 46-year-old male patient visited the Orthopedic Clinic of a local hospital (Yichun City Chinese Medicine Hospital, Yichun, China) in December 2014, complaining of severely painful swelling in his right thigh, which was preceded by a mild sprain that occurred whilst lifting heavy weights. Plain film radiographs (Kodak DirectView DR9000; Kodak, Rochester, NY, USA) performed at the local general hospital revealed a traumatic femoral fracture, which was treated by ORIF. The patient was discharged without any complications 2 weeks later, after the incision had healed. However, 20 days following surgery, gradual swelling and soreness with no obvious cause was observed around the incision. Eventually, the incision ruptured during squatting for bowel movement, which resulted in marked 
blood loss. The patient was referred to the Department of Orthopedics of The First Affiliated Hospital of Nanchang University for further treatment and was admitted in January 2015. A series of laboratory tests were performed shortly following admission: Blood hemoglobin levels were $66 \mathrm{~g} / 1$ (normal ranges, 120-160g/l), while inflammatory and tumor markers were within the normal ranges. Coagulant function was normal. The patient was diagnosed with severe hemorrhagic anemia, and required a transfusion of 12 units red blood cell suspensions, 18 units fresh frozen plasma, 13 units platelets and 20 units cryoprecipitate within 2 days of hospital admission. However, hemoglobin levels were only slightly increased to $\sim 80 \mathrm{~g} / 1$, and heavy bleeding due to the ruptured incision was observed. Plain radiographs revealed the presence of multiple radiopaque calcified bodies around the fracture (Fig. 1). In addition, they showed that the fracture had been considerably reduced following ORIF at the Yichun City Chinese Medicine Hospital (Fig 2A). A diagnosis of inter- or IH with calcification was considered; however, the differential diagnosis for the patient's condition was extensive, including parenchymal foreign bodies, myositis ossifications, aggressive fibromatosis and postoperative infection. CT (SOMATOM Definition AS CT Scanner; Siemens Healthcare, Henkestr, Germany) revealed an irregularly low-density mass shadow surrounding the femoral shaft in the anterolateral muscles of the right middle and distal thigh, and punctate calcification was observed (Fig. 2B). Following careful observation of the scans, cortical thinness and bone destruction was identified in the fracture ends (Fig. 2C). Enhanced CT demonstrated clear peripheral enhancement of the mass (Fig. 2D).

Based on the aforementioned findings, IH with pathological femoral fracture was suspected, and femoral artery arteriography was performed. Selective angiography (Axiom Artis BA DSA; Siemens Healthcare) demonstrated that the deep femoral artery was the feeder vessel of the IH (Fig. 3A and B); therefore, the patient underwent selective embolization of the deep femoral artery. Several days following treatment, the condition of the patient improved and the bleeding gradually stopped. The diagnosis of IH with pathological femoral fracture was confirmed based on these observations. The patient was discharged without complications 1 week subsequent to arterial embolization, when the patient's hemoglobin levels had reached $110 \mathrm{~g} / \mathrm{l}$. The patient was monitored by plain radiograph and CT scan during the 6 months of postoperative follow-up and by plain radiograph and MRI scan (Magnetom Trio Tim 3.0; Siemens AG, Munich, Germany) for 9 months after that; no sign of recurrence was observed and the patient returning to work. At the time of writing the fracture had healed. Rigorous monitoring of patients with pathological femoral fracture with IH is essential in order to detect any local recurrence or non-union of the femoral shaft.

\section{Discussion}

Hemangiomas are benign vascular tumors that are most likely to arise from the embryonic rest of unipotent angioblastic cells that have failed to develop into normal blood vessels. Hemangiomas account for $7-10 \%$ of all soft-tissue tumors $(6,7)$. IH is the most common type of deep soft-tissue hemangioma, one of the most common types of deep-seated soft tissue tumors, as well as the most common type of benign tumors of muscle (5).

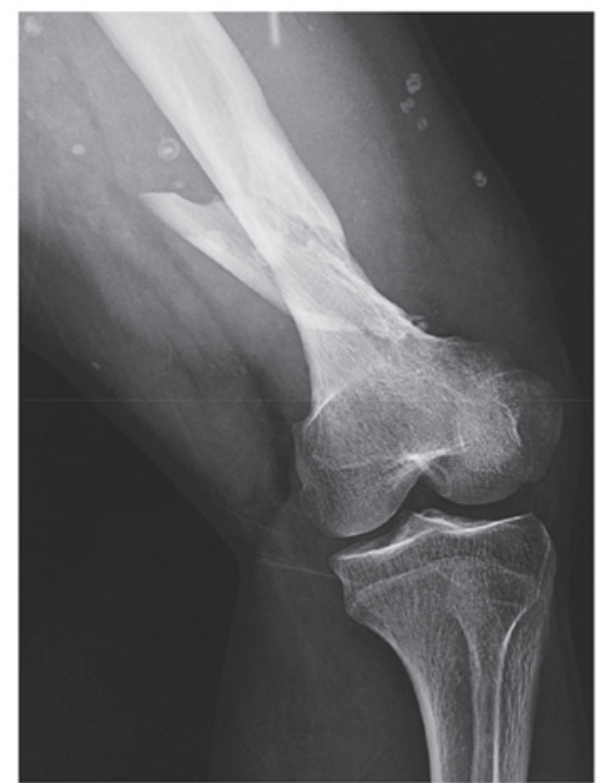

Figure 1. Preoperative plain radiographs revealing multiple radiopaque calcified bodies around a distal femoral fracture.

The majority of IHs originate in the skeletal muscle of the extremities, particularly the lower extremities. IH accounts for $<1 \%$ of all hemangiomas, and are primarily observed in young adults aged $<30$ years $(8,9)$. Chronic pain and novel mass development are the most common symptoms of IH (3). Pain has been recorded in $60 \%$ of cases, and is most frequent in long and narrow muscles. Pain is often aggravated by exercise of the involved muscles, since vascular dilation and increased regional blood flow leads to swelling and compressive pain $(10,11)$. A palpable tender mass is observed in the majority of IH cases. Large hemangiomas are identified by the detection of a vascular murmur or tremor upon palpation $(2,12)$. Superficial vein dilation may also be observed with cutaneous extension, and the tumor is generally long-standing, large and slowly-growing (4). Complications have been reported, such as nerve compression and compartment-like syndromes $(13,14)$. In general, IH is rarely considered in the differential diagnosis of musculoskeletal pain. The average duration of symptoms at initial presentation is 13 months, and so chronic extremity pain should increase suspicion for the presence of IH $(2,15)$. IH presenting with pathological fracture is extremely rare. It is essential that $\mathrm{IH}$ is considered in the differential diagnosis of parenchymal foreign bodies, myositis ossifications, aggressive fibromatosis and postoperative infection, when multiple radiopaque calcified bodies are observed around the fracture using plain radiographs. A diagnosis of IH is usually based on medical history, clinical examination and a combination of imaging studies.

The pathophysiology of these lesions has not been fully determined, but information may be gained from their underlying vascular nature. Isolated lesions are benign tumors that do not metastasize, but enlarge and involute over time (16). There are 3 histological types of hemangiomas: Capillary, cavernous and mixed. The majority of IHs are capillary, although, sometimes, mixed IHs may also have a dominant capillary component (17). The present case was a capillary $\mathrm{IH}$, according to the findings of the deep femoral artery 
A

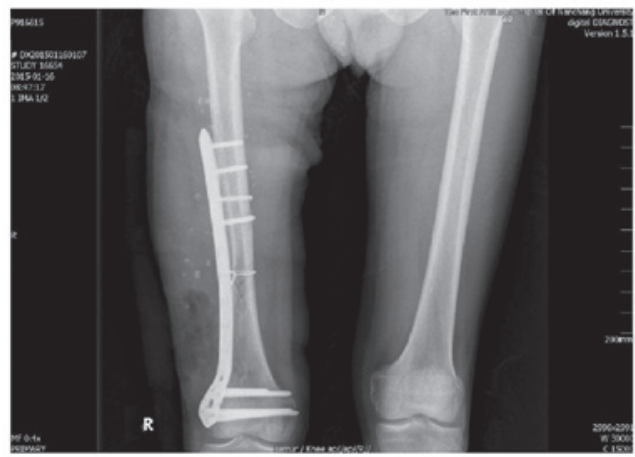

C

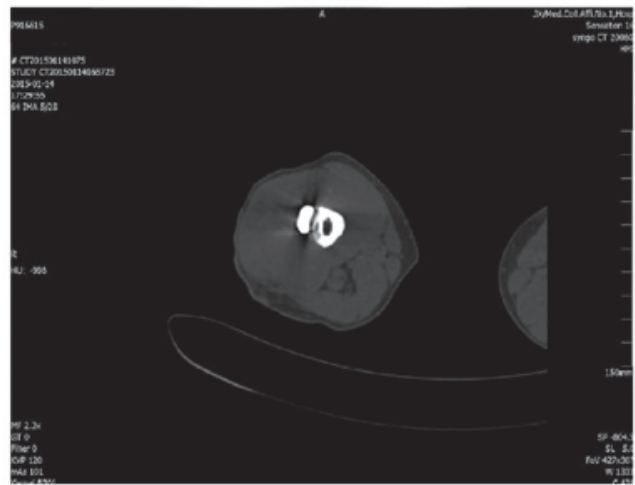

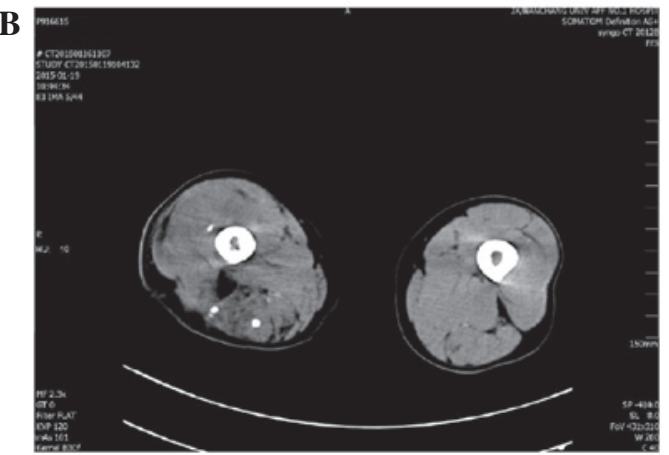

D

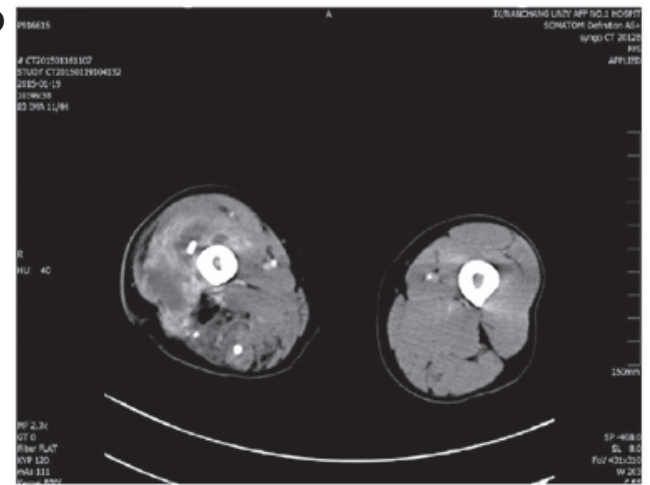

Figure 2. Postoperative imaging examinations. (A) Plain radiographs demonstrating that the femoral fracture was considerably reduced. (B) CT revealing an irregularly low-density mass shadow surrounding the femoral shaft in the anterolateral muscles of the right middle and distal thigh, with punctate calcification. (C) Cortical thinness and bone destruction of the fracture ends was observed using CT. (D) Enhanced CT revealed obvious peripheral enhancement of the mass. CT, computed tomography.

A

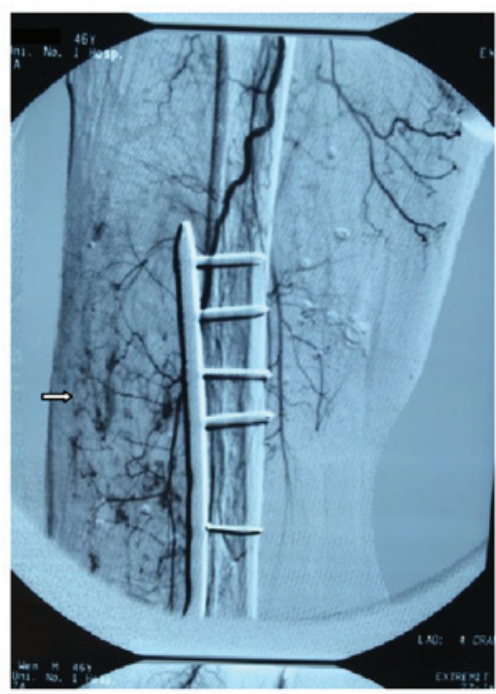

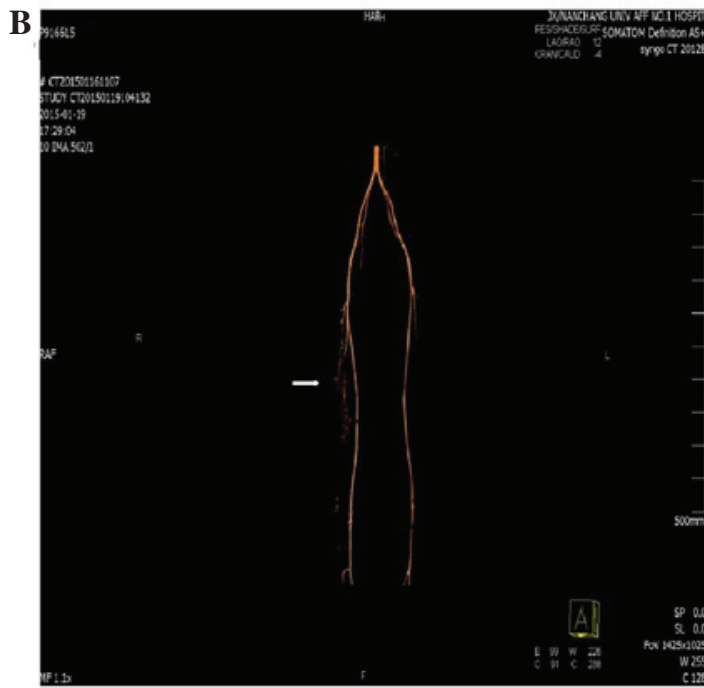

Figure 3. Selective artery arteriography and image reconstruction. (A and B) The deep femoral artery was identified as the feeder vessel of intramuscular hemangioma, and multiple messy capillaries were observed (white arrows).

arteriography. Pathology was not performed as it was not necessary for the diagnosis.

IHs are evaluated with plain radiographs, ultrasound, $\mathrm{CT}$, angiography and MRI. MRI is the preferred diagnostic modality, as it effectively differentiates between hemangiomas and malignant tumors without a biopsy. On T2-weighted images IH exhibits a high-signal intensity compared to skeletal muscle, which suggests the presence of dilated vascular spaces filled with stagnant blood. On T1-weighted images, $70 \%$ of IHs exhibit mildly hyperintense signals compared to skeletal muscle, while $30 \%$ are hyperintense $(4,18,19)$. On plain radiographs, rounded soft-tissue calcifications, known as 'phleboliths' or 'venous stones', may be observed in certain hemangiomas. Musculoskeletal ultrasound may reveal a mass, and is effective in the workup of IH. However, ultrasound does not reliably identify pathognomonic features of hemangiomas, but it does reveal abnormal Doppler flow patterns or features consistent with phleboliths (20). CT is helpful in excluding other types of masses from diagnosis, but is not always reliable in defining hemangiomas. Occasionally, angiography is required 
to demonstrate the fine vascular details of a hemangioma. This may be helpful in cases where embolization or surgical resection of complex lesions is considered. The majority of imaging modalities may identify certain features of $\mathrm{IH}$, and therefore biopsy is rarely required to rule out malignancy. However, if diagnostic uncertainty remains following clinical examination and imaging, open or needle biopsy is recommended $(18,21)$. A definitive diagnosis is only demonstrated following histopathological evaluation of the entire tumor.

Several treatment methods have been proposed for $\mathrm{IH}$, including conservative management, systemic corticosteroids, embolization, radiation, sclerotherapy and surgical excision. Each individual case requires thorough consideration of the unique characteristics of the lesion and degree of functional impairment. Conservative management is the preferred treatment option for nearly all isolated IHs; however, complete surgical excision of the lesion is the mainstay of IH treatment in the presence of rapid tumor growth, intense pain, risk of local skin necrosis, cosmetic or functional impairment or suspicion of malignancy . However, recurrence rates, particularly following incomplete surgical excision are between 18 and $61 \%(5,22)$. Surgical margins and tumor size have been reported as risk factors for recurrence. Selective embolization is feasible as large caliber vessels, which feed IH, are available for the procedure, as demonstrated by the present case. In addition, preoperative embolization is also performed for diffuse hemangiomas, when arteriography highlights tributary arteries of sufficient diameter (23). Additional treatments, such as corticosteroids, radiation and sclerotherapy, are available, but are rarely used due to side-effects and less successful long-term outcomes: Side-effects of corticosteroids include elevated blood pressure, changes to cholesterol levels, mood swings, acne and premature balding; side-effects of radiation include nausea, vomiting, inhibition of hematopoiesis and skin ulcers, and side-effects of sclerotherapy include tissue necrosis and pulmonary embolism $(2,24)$. Surgical excision is occasionally impractical; not all patients are recommended for surgery, and complex high-risk infiltrating lesions exist. In these cases, sclerotherapy, corticosteroids or radiation therapy may be beneficial.

In conclusion, the present study reports the case of a 46-year-old male patient who suffered from IH of the right thigh and presented with a pathological femoral fracture. Initially, the patient was misdiagnosed with traumatic femoral fracture, and routine open reduction and internal fixation were performed; however, based on the patient's medical history, plain radiographs, $\mathrm{CT}$ and artery arteriography, the diagnosis of pathological femoral fracture with IH was eventually made.In conclusion, a differential diagnosis of IH with pathological fracture should be considered when multiple radiopaque calcified bodies are observed around the fracture. In the present study, after 6 months of follow-ups, the patient was symptom-free, with CT demonstrating no evidence of recurrence. As the risk of tumor recurrence is high in IH, long-term follow-up is required.

\section{Acknowledgements}

This study was supported by the Gan-Po Talents Project 555 of Jiangxi Province and the Support Plan of Science and Technology Department of Jiangxi Province (grant no. 20112BBG70020).

\section{References}

1. Zide BM and Levine SM: Hemangioma update: Pearls from 30 years of treatment. Ann Plast Surg 69: 99-103, 2012.

2. Wierzbicki JM, Henderson JH, Scarborough MT, Bush CH, Reith JD and Clugston JR: Intramuscular hemangiomas. Sports Health 5: 448-454, 2013.

3. Kryzak TJ Jr and DeGroot H III: Adult onset flatfoot associated with an intramuscular hemangioma of the posterior tibialis muscle. Orthopedics 31: 280, 2008.

4. Griffin N, Khan N, Thomas JM, Fisher C and Moskovic EC: The radiological manifestations of intramuscular haemangiomas in adults: Magnetic resonance imaging, computed tomography and ultrasound appearances. Skeletal Radiol 36: 1051-1059, 2007.

5. Tang P, Hornicek FJ, Gebhardt MC, Cates J and Mankin HJ: Surgical treatment of hemangiomas of soft tissue. Clin Orthop Relat Res 399: 205-210, 2002.

6. Jahn H and Nissen HM: Haemangioma of the urinary tract: Review of the literature. Br J Urol 68: 113-117, 1991.

7. Rigopoulou A and Saifuddin A: Intraosseous hemangioma of the appendicular skeleton: Imaging features of 15 cases and a review of the literature. Skeletal Radiol 41: 1525-1536, 2012.

8. Brown RA, Crichton K and Malouf GM: Intramuscular haemangioma of the thigh in a basketball player. Br J Sports Med 38: 346-348, 2004.

9. Wu JL, Wu CC, Wang SJ, Chen YJ, Huang GS and Wu SS: Imaging strategies in intramuscular haemangiomas: An analysis of 20 cases. Int Orthop 31: 569-575, 2007.

10. Wild AT, Raab P and Krauspe R: Hemangioma of skeletal muscle. Arch Orthop Trauma Surg 120: 139-143, 2000.

11. Ranero-Juárez AG, Rosales-Galindo VM, León-Takahashi AM, Arenas-Guzmán R and García C: Intramuscular hemangiomas of the extremities: Report of six cases. Int J Dermatol 48: 875-878, 2009.

12. Jamshidi K, Jafari D, Ramezan Shirazi M, Pahlevansabagh A and Shoushtaryzadeh T: An unusual presentation of ossified intramuscular hemangioma: A case report. Acta Med Iran 52:319-322, 2014.

13. Downey-Carmona FJ, González-Herranz P, De La Fuente-González C and Castro M: Acute compartment syndrome of the foot caused by a hemangioma. J Foot Ankle Surg 45: 52-55, 2006.

14. Nazzi V, Messina G, Dones I, Ferroli P and Broggi G: Surgical removal of intramuscular arteriovenous hemangioma of the upper left forearm compressingradial nerve branches. J Neurosurg 108: 808-811, 2008.

15. Wisniewski SJ, Newcomer K and Stanson AW: Intramuscular hemangioma of the foot: A diagnostic dilemma. Med Sci Sports Exerc 37: 1655-1657, 2005.

16. Henderson J, Wierzbicki J, Clugston J and Patel N: Ten years of lower leg pain: Intramuscular hemangioma. Med Sci Sports Exer 42: 5, 2010.

17. Lee JK and Lim SC: Intramuscular hemangiomas of the mylohyoid and sternocleidomastoid muscle. Auris Nasus Larynx 32: 323-327, 2005.

18. Buetow PC, Kransdorf MJ, Moser RP Jr, Jelinek JS and Berrey BH: Radiologic appearance of intramuscular hemangioma with emphasis on MR imaging. AJR Am J Roentgenol 154: 563-567, 1990.

19. Teo EL, Strouse PJ and Hernandez RJ: MR imaging differentiation of soft tissue hemangiomas from malignant soft-tissue masses. AJR Am J Roentgenol 174: 1623-1628, 2000.

20. McNeill TW, Chan GE, Chapek V and Ray RD: The value of angiography in the surgical management of deep hemangiomas. Clin Orthop Relat Res 101: 176-181, 1974.

21. Greenspan A, McGahan JP, Vogelsang P and Szabo RM: Imaging strategies in the evaluation of soft-tissue hemangiomas of the extremities: Correlation of the findings of plain radiography, angiography, CT, MRI and ultrasonography in 12 histologically proven cases. Skeletal Radiol 21: 11-18, 1992.

22. Bella G, Manivel J, Thompson RC Jr, Clohisy DR and Cheng EY: Intramuscular hemangioma: Recurrence risk related to surgical margins. Clin Orthop Relat Res 459: 186-191, 2007.

23. Holzapfel BM, Geitner U, Diebold J, Glaser C, Jansson V and Dürr HR: Synovial hemangioma of the knee joint with cystic invasion of the femur: A case report and review of the literature. Arch Orthop Trauma Surg 129: 143-148, 2009.

24. Zide BM and Levine SM: Hemangioma update: Pearls from 30 years of treatment. Ann Plast Surg 69: 99-103, 2012. 